\title{
Path Optimization for Rhombic-Like Vehicles: An Approach Based on Rigid Body Dynamics
}

\author{
Daniel Fonte*, Filipe Valente*, Alberto Vale*, Isabel Ribeiro ${ }^{\dagger}$ \\ *Instituto de Plasmas e Fusão Nuclear - Laboratório Associado - Instituto Superior Técnico \\ ${ }^{\dagger}$ Institute for Systems and Robotics - Instituto Superior Técnico \\ Av. Rovisco Pais 1, 1049-001, Lisboa - Portugal \\ Email: \{daniel.fonte, filipe.valente, avale\}@ipfn.ist.utl.pt, mir@isr.ist.utl.pt
}

\begin{abstract}
This paper proposes a new path optimization method to improve feasibility and optimality of rough collisionfree paths provided by global planners. Inspired on the dynamics of rigid bodies, this method redefines the elastic bands framework to explicitly handle vehicle geometric constraints and explore the high maneuvering ability of rhombic vehicles. The proposed method was tested as a post optimization technique on global path solutions planned for a large transporter that will operate in the International Thermonuclear Experimental Reactor (ITER). Presented results show the proficiency of this method on handling feasible and reliable paths in cluttered scenarios such as those in ITER.
\end{abstract}

\section{INTRODUCTION}

The International Thermonuclear Experimental Reactor (ITER) is a world project on fusion research that will constitute the largest tokamak-based research facility. Besides the major scientific objective of a viable use of fusion energy, ITER aims to demonstrate that the future fusion power plants can be safely and effectively maintained. After the start of machine operation, its main components become activated preventing human presence in certain areas and therefore, the ITER maintenance system will mostly depend on reliable and robust Remote Handling (RH) systems.

The Cask and Plug Remote Handling System (CPRHS) is a large transporter, which will provide the means to transfer in-vessel components and $\mathrm{RH}$ tools between the Tokamak Building (TB) and the Hot Cell Building (HCB). Each CPRHS is composed by three subsystems: the cask envelope, the pallet and the Cask Transfer System (CTS), as depicted in Fig. 1-left. The largest CPRHS has dimensions $8.5 \mathrm{~m} \times 2.62 \mathrm{~m} \times 3.62 \mathrm{~m}$ (length $\mathrm{x}$ width $\times$ height) and can reach a maximum weight, when loaded, of $\sim 100 T$. The CTS acts as a mobile robot and can move independently from the cask and pallet. This mobile platform was conceived with a rhombic configuration (hence the rhombi-like vehicle moniker or simply rhombic vehicle) consisting on two pairs (one for spare purposes) of drivable and steerable wheels positioned on the front and rear of the vehicle and two swivel

Work supported by the grant F4E-GRT-276-01(MS-RH) funded by the European Joint Undertaking for ITER and the Development of Fusion Energy (F4E-Fusion for Energy) and by FCT in the frame of the Contract of Associate Laboratories of Instituto de Plasmas e Fusão Nuclear/IST and Institute for Systems and Robotics/IST. The views expressed in this publication are the sole responsibility of the authors. F4E is not liable for the use which might be made of the information in this publication. wheels on the sides (see Fig.1-right). This kinematic configuration was proposed in [7] to endow the transporter with an high maneuvering ability and flexibility, key traits when considering the cluttered nature of the ITER environments.

The CPRHS should move along optimized prescribed paths according to schedule or unscheduled maintenance operations. Considering the large dimensions of the vehicle and the cluttered nature of the environments, each planned path shall satisfy the following criteria: (1) maximize the clearance to the obstacles to reduce the risk of collision; (2) minimize the distance traveled to prevent the need to recharge the on-board batteries during the journeys; (3) guarantee smooth transitions to avoid jerky motions and reduce the number of maneuvers.

The use of motion planning techniques [6], [14] appears as a powerful tool to plan and analyze the required motions for these missions. Regardless of their robustness to solve specified motion queries, these planning techniques can perform poorly when considering the quality and feasibility of the planned solutions. Hence, seminal forms of general motion planning techniques are commonly coupled with optimization tools as an attempt to close the gap between planning and execution phases. For instance, [15] introduced the elastic bands as a combined planning and control methodology to dynamically deform paths while allowing real time obstacle avoidance. The idea of dynamically deform paths evolved and met important progresses [8], [3] and provided extensions to higher planning instances [5]. Other approaches exist that attempt to increase the planner reliability by embedding realistic simulators on the planner, [11]. Post optimization techniques are also employed to improve path quality and support a posteriori tracking phases, [10].

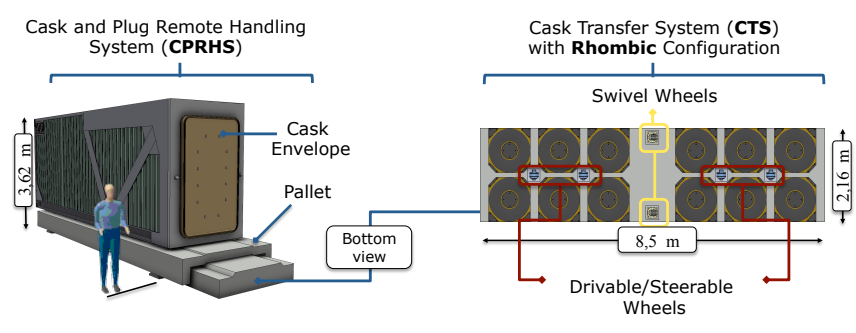

Fig. 1. Left: schematic view of the CPRHS. Right: the CTS with its rhombic configuration. 
The motion planning problem for the CPRHS rhombic vehicle was first addressed in [1] by a planning methodology, which granted the generation of feasible and optimized trajectories in agreement with the above specified criteria. The inbuilt path optimization module followed closely the elastic bands approach proposed in [15]. However, this methodology was compliant with a line guidance requirement entailing that both vehicle wheels should follow the same physical path and therefore, the inherent rhombic flexibility was only partially explored. Fig.2 illustrates part of the scenario in TB of ITER where a CPRHS, acting as a rescue vehicle, has to dock in a Vacuum Vessel Port Cell (VVPC) where another CPRHS is parked. For the particular case where both wheels are constrained to follow the same path (center), no solution is found. However, the use of independent references for the wheels would simplify the motion problem (right). The achievement of this solution requires the use of dedicated motion planning techniques, in particular, the employment of a efficient path optimization method capable of handling the high maneuvering ability of the rhombic vehicle.

Accepting as accomplished the motion planning or path planning problem and drawing inspiration in this ITER case study, this paper proposes a novel path optimization method that acts on rough paths provided by global planners like the Rapidly-Exploring Random Tree (RRT), [13], or the Probabilistic Roadmap Method (PRM) [16]. The proposed method redefines the elastic bands concept, [15], to evade the common approach that formulates paths as particle-systems. Inspired on the rigid body dynamics, consecutive poses along the rough path previously referred are treated as rigid bodies that are repealed from obstacles through external forces, improving path clearance. Additional interactions provide path connectivity and guarantee smooth transitions between vehicle poses. This formulation allow to explicitly consider the vehicle geometry during the optimization and fully profit from the high maneuverability of rhombic vehicles.

The paper is organized as follows. Section II reviews some notions on rigid body dynamics and describes the proposed method for path optimization. The remaining sections lay out the obtained simulation results (Section III) and outline directions for future work (Section IV).

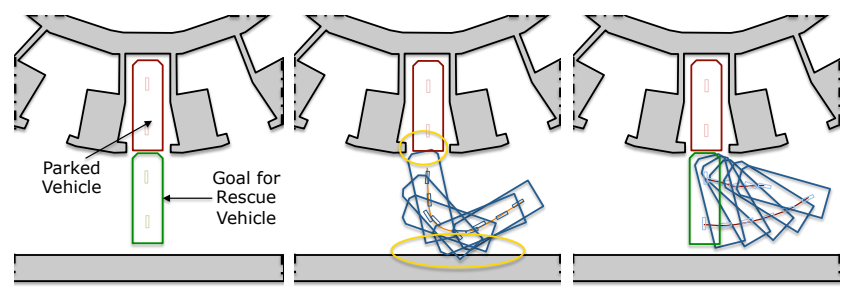

Fig. 2. Rescue scenario in the TB: the green rescue vehicle must assist the red parked vehicle (left); path solution for the same path for both wheels (center); independent paths for each wheel (right).

\section{Path Optimization Based on Rigid Body DYNAMICS}

\section{A. Dynamics of Rigid Bodies}

For this work's purposes, the use of rigid body dynamics is restricted to the case of general plane motion, i.e., the particles composing the rigid body move in parallel planes and their motion is neither characterized by pure rotational nor pure translational movements. Therefore, angular variables, such as moments and angular velocities, are scalar quantities. In the sequel, bold-face type is used to represent vector quantities.

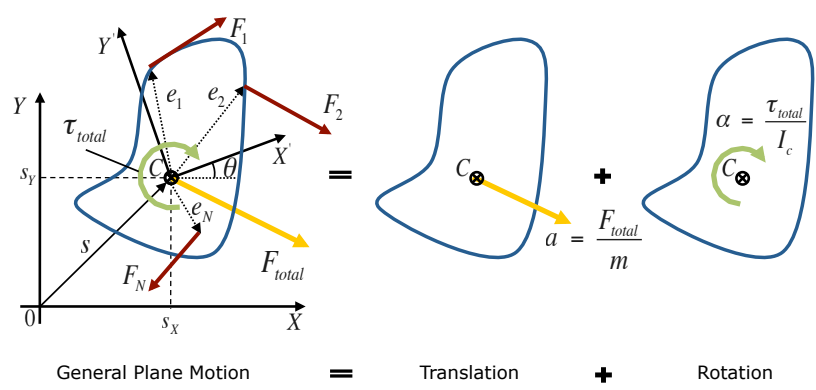

Fig. 3. Kinetics of rigid bodies: the general plane motion of a rigid body can be decomposed on a translation and a rotation about $C$.

Consider that a rigid body with a Center of Mass (CoM) denoted by $C$, as represented in Fig.3, is acted by $N$ external forces, $\boldsymbol{F}_{\boldsymbol{n}}$, with $\{n=1, \cdots, N\}$. Following the Newton's Second Law and taking the rigid body as a system of particles, the dynamics of $C$, with respect to the inertial frame $O X Y$, is given by

$$
\boldsymbol{F}_{\text {total }}=\sum_{n=1}^{N} \boldsymbol{F}_{\boldsymbol{n}}=m \boldsymbol{a},
$$

where $m$ is the mass of the body and $\boldsymbol{a}$ is the linear acceleration of $C$. The dynamics of the rigid body motion relative to the its body frame, $C X^{\prime} Y^{\prime}$, is given by

$$
\boldsymbol{\tau}_{\text {total }}=\sum_{n=1}^{N} \boldsymbol{F}_{\boldsymbol{n}} \times \boldsymbol{e}_{\boldsymbol{n}}=I_{C} \alpha,
$$

which entails that the resultant torque about $C, \tau_{\text {total }}$, is a vector with the direction of the angular acceleration, $\alpha$, and magnitude $I_{C} \alpha$. In (2), $I_{C}$ is the moment of inertia around the perpendicular axis passing through $C$, whereas $\boldsymbol{e}_{\boldsymbol{n}}$ corresponds to the position vector of $\boldsymbol{F}_{\boldsymbol{n}}$ relative to the reference frame $C X^{\prime} Y^{\prime}$. For the case of uniformly accelerated motion, which will be adopted in this formulation, $\boldsymbol{a}$ and $\alpha$ assume constant values over time.

As it is represented in Fig. 3 and, from the kinetics viewpoint, the general plane motion of the rigid body can be decomposed as the combination of a translation with linear acceleration, $\boldsymbol{a}$, and a rotation about $C$ with angular acceleration, $\alpha$, given by (1) and (2), respectively. The linear, $\boldsymbol{v}$, and angular, $\omega$, velocities of the rigid body's CoM can be obtained through integration of $\boldsymbol{a}$ and $\alpha$ over time, $t$, as follows,

$$
v=v^{0}+a t
$$




$$
\omega=\omega^{0}+\alpha t
$$

where $v^{0}$ and $\omega^{0}$ are the initial linear and angular velocities.

The position, $s$, and orientation, $\theta$, of the rigid body can be accessed through the integration of (3) and (4), yielding

$$
\begin{aligned}
& s=s^{0}+\boldsymbol{v}^{0} t+\frac{1}{2} \boldsymbol{a} t^{2} \\
& \theta=\theta^{0}+\omega^{0} t+\frac{1}{2} \alpha t^{2},
\end{aligned}
$$

where $s^{0}$ and $\theta^{0}$ are the initial position and orientation of the rigid body's CoM. Equations (1) - (6) completely describe the general plane motion of a rigid body, relating displacement, velocity and acceleration to the external forces, which are the cause of motion.

\section{B. Path Optimization based on Rigid Body Dynamics}

The path optimization proposed in this paper works as a post processing method, which, based on a deformation process, refines and improves the quality of a rough solution path provided by a planner. This rough path, which defines the input for the optimization process, is consider to be a set of collision-free motions connecting the queried start, $\boldsymbol{q}_{\boldsymbol{s}}$, and the goal, $\boldsymbol{q}_{\boldsymbol{g}}$, vehicle configurations (see Fig. 4-left). From this time forth the rough path will be referred to as query path.

In the path optimization process, each of the consecutive vehicle poses that form the query path is treated as a rigid body that is connected with its adjacent poses like a convoy through internal interactions and subjected to externalrepulsive forces produced by obstacles in its vicinity. Hence, the path optimization becomes a path deformation problem, which relies on the principles of rigid body dynamics to iteratively simulate the evolution of each pose on the optimization process. In particular, it is proposed to subject each vehicle pose in the query path to two types of efforts:

- Internal efforts - Consecutive poses are kept connected through virtual elastic and torsional springs, which simulate the Hooke's elasticity concept and originate elastic forces and torsional torques. These efforts guarantee smoothness on deformation and help to shorten the path;

- External efforts - Repulsive forces repel the rigid poses from obstacles, acting as a collision avoidance feature. Moreover, force eccentricity originates repulsive rotating torques, which readapt poses orientation maximizing clearance over the obstacles.

The novel method proposed in this paper disregards line guidance approaches as the ones proposed in [1] with the resulting optimized path defining an independent reference for each vehicle wheel. Loosely following the elastic bands concept proposed by Quinlan and Khatib in [15], this method, by considering each vehicle pose as a rigid body, enables the path deformation to explicitly consider the vehicle geometry and exploit the rhombic vehicle nature, issues considered here as unattended on similar studies.

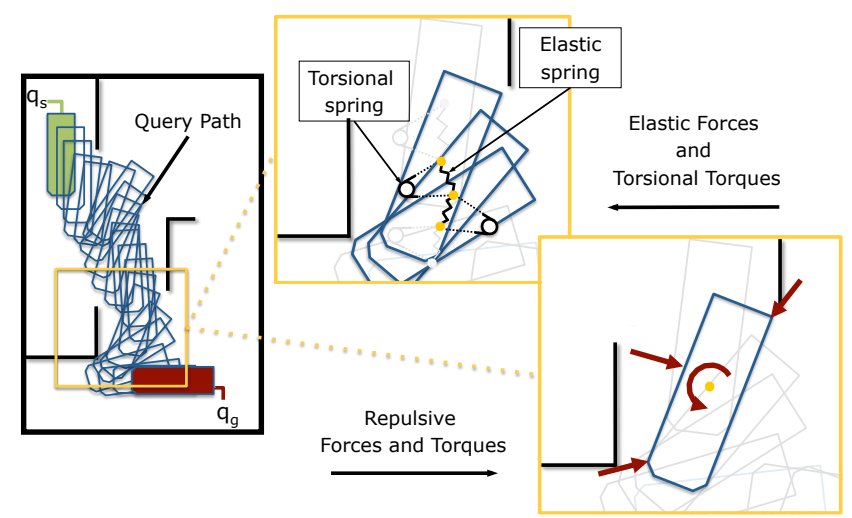

Fig. 4. Optimization based on the rigid body dynamics: the elastic forces and torsional torques help to smooth and shorten the path while the repulsive forces and torques maximize clearance from obstacles.

\section{Implementation}

Let $j=\{1, \ldots, J\}$ be the index of the consecutive vehicle poses composing the query path, each defined by a configuration vector

$$
\boldsymbol{q}_{\boldsymbol{j}}=\left[\begin{array}{c}
\boldsymbol{s}_{\boldsymbol{j}} \\
\theta_{j}
\end{array}\right]
$$

where $s_{j}$ and $\theta_{j}$ denote the position and the orientation of the pose $\boldsymbol{q}_{j}$ relative to a fixed reference frame, respectively. It is stated that $\boldsymbol{q}_{\mathbf{1}}=\boldsymbol{q}_{\boldsymbol{s}}$ and $\boldsymbol{q}_{\boldsymbol{J}}=\boldsymbol{q}_{\boldsymbol{g}}$.

The elastic force, $\boldsymbol{F}_{\boldsymbol{E}}$, and the torsional torque, $\tau_{T}$, evaluated for the vehicle pose at $\boldsymbol{q}_{\boldsymbol{j}}$ are:

$$
\begin{aligned}
\boldsymbol{F}_{\boldsymbol{E}}\left(\boldsymbol{q}_{\boldsymbol{j}}\right) & =K_{E} \cdot\left[\left(\boldsymbol{s}_{\boldsymbol{j}+\mathbf{1}}-\boldsymbol{s}_{\boldsymbol{j}}\right)+\left(\boldsymbol{s}_{\boldsymbol{j}-\mathbf{1}}-\boldsymbol{s}_{\boldsymbol{j}}\right)\right] \\
\tau_{T}\left(\boldsymbol{q}_{\boldsymbol{j}}\right) & =K_{T} \cdot\left[\left(\theta_{j+1}-\theta_{j}\right)+\left(\theta_{j-1}-\theta_{j}\right)\right],
\end{aligned}
$$

where $K_{E}$, the elasticity gain and, $K_{T}$, the torsional gain, control the elastic and torsional avoidance behavior on the path deformation, respectively.

The evaluation of the external efforts due to obstacle proximity relies on a heuristic-based collision detector module, which is capable of determining the set of i-nearest Obstacle Points (OPs) to each sampled pose $\boldsymbol{q}_{j}$. The overall procedure to handle the evaluation of the repulsive forces and torques is illustrated in Fig. 5 and can be described as follows:

1) Let $i=\{1, \ldots, I\}$ denote the index of the $i$-th OP relative to a specific pose $\boldsymbol{q}_{j}$. Let $\boldsymbol{u}_{j, i}$ be the vector

$$
u_{j, i}=V_{j, i}-O_{j, i}
$$

taken from each $\mathrm{OP}, \boldsymbol{O}_{j, i}$, and the corresponding vehicle nearest point, $\boldsymbol{V}_{\boldsymbol{j}, \boldsymbol{i}}$.

2) To improve clearance during path deformation, distance-dependent repulsive forces are defined, where each pair of points $\left(\boldsymbol{O}_{j, i}, \boldsymbol{V}_{j, i}\right)$ determines a repulsive contribution. For a specific vehicle pose, $\boldsymbol{q}_{j}$, the repulsive contributions are defined as

$$
\boldsymbol{r}_{\boldsymbol{j}, \boldsymbol{i}}=\frac{\boldsymbol{u}_{\boldsymbol{j}, \boldsymbol{i}}}{\left\|\boldsymbol{u}_{\boldsymbol{j}, \boldsymbol{i}}\right\|} \cdot f\left(\left\|\boldsymbol{u}_{\boldsymbol{j}, \boldsymbol{i}}\right\|\right)
$$

where,

$$
f\left(\left\|\boldsymbol{u}_{j, i}\right\|\right)=\max \left(0, F_{\max }-\frac{F_{\max }}{d_{\max }} \cdot\left\|\boldsymbol{u}_{\boldsymbol{j}, \boldsymbol{i}}\right\|\right) .
$$


In (12), a maximum allowable magnitude, $F_{\max }$, is assigned to avoid outsized values in the close vicinity of the obstacles. $d_{\max }$ denotes the distance up to which the repulsive force is applied.

3) For each pose $\boldsymbol{q}_{\boldsymbol{j}}$, the total repulsive force is defined as

$$
\boldsymbol{F}_{\boldsymbol{R}}\left(\boldsymbol{q}_{\boldsymbol{j}}\right)=\sum_{i=1}^{I} \boldsymbol{r}_{\boldsymbol{j}, \boldsymbol{i}}
$$

Using (2), the net repulsive torque around the $j$-th pose CoM is defined as

$$
\tau_{R}\left(\boldsymbol{q}_{\boldsymbol{j}}\right)=\sum_{i=1}^{I} \boldsymbol{r}_{\boldsymbol{j}, \boldsymbol{i}} \times \boldsymbol{e}_{\boldsymbol{j}, \boldsymbol{i}}
$$

The repulsive and elastic forces are combined on a total force contribution as,

$$
\boldsymbol{F}_{\text {total }}\left(\boldsymbol{q}_{j}\right)=\boldsymbol{F}_{\boldsymbol{R}}\left(\boldsymbol{q}_{j}\right)+\boldsymbol{F}_{\boldsymbol{E}}\left(\boldsymbol{q}_{\boldsymbol{j}}\right) .
$$

Similar approach is valid for the torsional and repulsive torques acting on each pose $\boldsymbol{q}_{j}$. This leads to the definition of a net torque expressed as,

$$
\tau_{\text {total }}\left(\boldsymbol{q}_{\boldsymbol{j}}\right)=\tau_{T}\left(\boldsymbol{q}_{\boldsymbol{j}}\right)+\tau_{R}\left(\boldsymbol{q}_{\boldsymbol{j}}\right) .
$$

The total force and the total torque are represented in Fig.5.

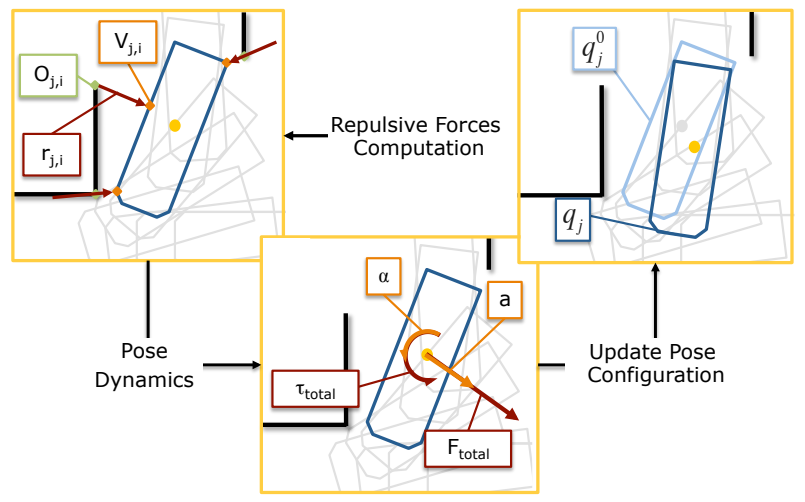

Fig. 5. Path optimization flow based on the rigid body dynamics.

Once determined the efforts acting on each pose, the ensued motion is evaluated through the principles of rigid body dynamics, as explained in Section II-A. Equations (1) and (2), are rewritten as

$$
\begin{gathered}
\boldsymbol{a}_{\boldsymbol{j}}=\frac{\boldsymbol{F}_{\text {total }}\left(\boldsymbol{q}_{\boldsymbol{j}}\right)}{m}-K_{D} \boldsymbol{v}_{\boldsymbol{j}} \\
\alpha_{j}=\frac{\tau_{\text {total }}\left(\boldsymbol{q}_{\boldsymbol{j}}\right)}{I_{C}}-K_{D} \omega_{j},
\end{gathered}
$$

which provide the linear and angular accelerations for a specific pose $\boldsymbol{q}_{\boldsymbol{j}}$. The last term in the right hand side of (7) and (8) represent damping effects introduced to reduce the oscillatory motion during path deformation. They are controlled through $K_{D}$, herein set equally for both the translational and the rotational motion components. Notice that both $m$ and $I_{C}$ in (17)-(18), do not refer to real vehicle parameters but rather to simple scalars determining the resistance of each pose to change its configuration.

From a starting configuration in the query path, $\boldsymbol{q}_{j}$, this pose is updated iteratively according to the set of equations
(3)-(6), where the referred initial conditions are the previous iterated pose in this process. The stopping criteria is defined by setting a maximum number of iterations.

\section{Numerical Approximation}

To solve the problem stated above the use of numerical integration methods is required. The simpler Euler integration method tends to be numerically unstable and less accurate for small rates [9], meaning that the path may oscillate widely and not reaching a stable configuration. This paper proposes the use of the Leapfrog method, which is a modified version of the Verlet method [2] and is nicely discussed in [12]. The Leapfrog method is commonly used to integrate Newton's equations of motion offering a greater stability when compared to the simpler Euler method.

Assume that the time discretization interval is $\Delta t$ and represent as $v_{j}^{k \Delta t}=v_{j}^{k}$ the value of the linear velocity of the pose iterated from $\boldsymbol{q}_{j}$ at time instant $k \Delta t$. According to the flow diagram represented in Figure 6, the Leapfrog algorithm can be described for the previous stated approach as follows: (a) the linear, $\boldsymbol{a}_{j}^{\boldsymbol{k}}$, and the angular, $\alpha_{j}^{k}$, accelerations are evaluated at a given time step $k$ using (17) and (18) with $v_{j}$ and $\omega_{j}$ given by (24) and (25) and $\boldsymbol{F}_{\text {total }}$ and $\tau_{\text {total }}$ evaluated at $\boldsymbol{q}_{j}^{\boldsymbol{k}}$; (b) the corresponding velocities are calculated for the next "half step" (i.e., $k+\frac{1}{2}$ ) as

$$
\begin{gathered}
\boldsymbol{v}_{\boldsymbol{j}}^{\boldsymbol{k}+\mathbf{1 / 2}}=\boldsymbol{v}_{\boldsymbol{j}}^{\boldsymbol{k - 1 / 2}}+\Delta t \boldsymbol{a}_{\boldsymbol{j}}^{\boldsymbol{k}}, \\
\omega_{j}^{k+1 / 2}=\omega_{j}^{k-1 / 2}+\Delta t \alpha_{j}^{k}
\end{gathered}
$$

(c) accordingly, the iterated configuration of the pose $\boldsymbol{q}_{j}$ at time instant $k+1$ is then updated from the iterated configuration of the pose at time instant $k$ as

$$
\boldsymbol{q}_{\boldsymbol{j}}^{\boldsymbol{k}+\mathbf{1}}=\left[\begin{array}{c}
\boldsymbol{s}_{\boldsymbol{j}}^{\boldsymbol{k}+\mathbf{1}} \\
\theta_{j}^{k+1}
\end{array}\right]=\boldsymbol{q}_{\boldsymbol{j}}^{\boldsymbol{k}}+\Delta t\left[\begin{array}{c}
\boldsymbol{v}_{\boldsymbol{j}}^{\boldsymbol{k}+\mathbf{1} / \mathbf{2}} \\
\omega_{j}^{k+1 / 2}
\end{array}\right]
$$

for $k \geq 0$. Note that $\boldsymbol{q}_{\boldsymbol{j}}^{\mathbf{0}}=\boldsymbol{q}_{\boldsymbol{j}}$, the pose at the query path.

It remains an issue how to evaluate the velocity at the next half-step when only the starting conditions are given. To get the calculation started and, as suggested in [12], the following simple approximation is used

$$
\begin{aligned}
& \boldsymbol{v}_{\boldsymbol{j}}^{\mathbf{1 / 2}}=\boldsymbol{v}_{\boldsymbol{j}}^{\mathbf{0}}+\frac{\Delta t}{2} \boldsymbol{a}_{\boldsymbol{j}}^{\mathbf{0}} \\
& \omega_{j}^{1 / 2}=\omega_{j}^{0}+\frac{\Delta t}{2} \alpha_{j}^{0} .
\end{aligned}
$$

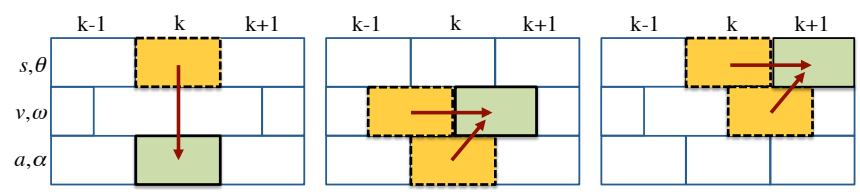

Fig. 6. Flow diagram of the Leapfrog algorithm: evaluation of $\boldsymbol{a}_{j}^{\boldsymbol{k}}$ and $\alpha_{j}^{k}$ (left); evaluation of $\boldsymbol{v}_{\boldsymbol{j}}^{\boldsymbol{k}+\mathbf{1 / 2}}$ and $\omega_{j}^{k+1 / 2}$ (center); update of $\boldsymbol{q}_{\boldsymbol{j}}^{\boldsymbol{k}+\mathbf{1}}$ (right). 
The velocities at any given instant $k$ are interpolated with

$$
\begin{aligned}
\boldsymbol{v}_{j}^{k} & =\frac{1}{2}\left[\boldsymbol{v}_{j}^{\boldsymbol{k}+\mathbf{1 / 2}}+\boldsymbol{v}_{j}^{\boldsymbol{k}-\mathbf{1} / \mathbf{2}}\right] \\
\omega_{j}^{k} & =\frac{1}{2}\left[\omega_{j}^{k+1 / 2}+\omega_{j}^{k-1 / 2}\right] .
\end{aligned}
$$

\section{Simulation Results}

The experiments presented in this paper were performed in the Trajectory Evaluator and Simulator (TES), a software tool developed in the MATLAB platform under the grant F4E2008-GRT-016 (MS-RH), which incorporates a Bidirectional RRT planner [13], to compute the initial query path and the proposed path optimization. Three measurements (clearance, length and smoothness) were defined to assess the performance of the obtained optimized paths.

Clearance: the clearance for a given pose configuration is defined as the minimum distance between the vehicle at that pose and the obstacles, i.e,

$$
C\left(\boldsymbol{q}_{\boldsymbol{j}}\right)=\min \left(\left\|\boldsymbol{u}_{\boldsymbol{j}, \boldsymbol{i}}\right\|\right) .
$$

The total and average clearance, $C$ and $\mu_{C}$, are defined for a path with $J$ samples as,

$$
C=\sum_{j=1}^{J} C\left(\boldsymbol{q}_{j}\right), \quad \mu_{C}=\frac{C}{J} .
$$

As proposed in [10], the bad clearance $(B C)$ evaluated over a path is defined as

$$
B C=\sum_{j=1}^{J} C_{b a d}\left(\boldsymbol{q}_{j}\right),
$$

where $C_{b a d}\left(\boldsymbol{q}_{\boldsymbol{j}}\right)=C_{\text {min }}-C\left(\boldsymbol{q}_{\boldsymbol{j}}\right)$, if $C\left(\boldsymbol{q}_{\boldsymbol{j}}\right)<C_{\text {min }}$, otherwise $C_{b a d}$ equals zero. In this study and following ITER specifications, $C_{\min }$ is set to 0.3 meters.

Length: path length is defined as the distance travelled by the vehicle center throughout the path. This measure is decomposed in a translational component,

$$
L T=\sum_{j=1}^{J-1}\left\|\left(\boldsymbol{s}_{\boldsymbol{j}+\mathbf{1}}-\boldsymbol{s}_{\boldsymbol{j}}\right)\right\|,
$$

and in a rotational component,

$$
L R=\sum_{j=1}^{J-1}\left\|\chi\left(\theta_{j+1}-\theta_{j}\right)\right\|,
$$

where $\chi$ represents an operator that normalizes the twisting angle in $[-\pi, \pi]$.

Smoothness: smoothness can be quantified resorting to the path derivate. Using a simple forward finite difference to approximate the derivate in pose $\boldsymbol{q}_{\boldsymbol{j}}$ both for the translational and the rotational components, as

$$
S T\left(\boldsymbol{q}_{\boldsymbol{j}}\right)=\left\|\boldsymbol{s}_{\boldsymbol{j}+\mathbf{1}}-\boldsymbol{s}_{\boldsymbol{j}}\right\|, \quad S R\left(\boldsymbol{q}_{\boldsymbol{j}}\right)=\left\|\chi\left(\theta_{j+1}-\theta_{j}\right)\right\|,
$$

the average and standard deviation for the translational and rotational smoothness of the path, denoted as $\mu_{S T}, \mu_{S R}, \sigma_{S T}$ and $\sigma_{S R}$, are evaluated.

The proficiency of the proposed optimization method is herein analyzed over three different path queries in different planning scenarios. Both the query and the optimization phases used the following dimensions for the rhombic vehicle: $8.5 \mathrm{~m} \times 2.62 \mathrm{~m}$ (length(l) $\mathrm{x}$ width $(\mathrm{w})$ ) for the Scenarios I and II and $5.5 \mathrm{~m} \times 2.62 \mathrm{~m}$ for the Scenario III. These experiments used $m=1$, with the moment of inertia defined as $\frac{m}{2} \cdot\left(w^{2}+l^{2}\right)$. The remanning constants were tuned according to the characteristics of each scenario.

Scenario I: the queried motion consists in a simple and general operation between two arbitrary configurations on a illustrative environment as the one presented in Fig.4. The Fig.7-left depicts the query path obtained using the RRT algorithm. This is a rough solution that it is not

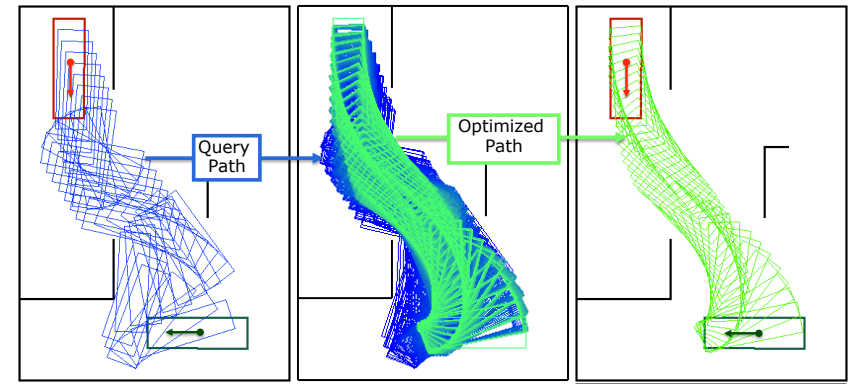

Fig. 7. Left: query path. Center: path optimization process; The path is gradually deformed from the poses represented in blue to the ones represented in green. Right: final optimized path.

feasible for execution due to path discontinuity and lack of path clearance. Fig. 7-center illustrates the process of path optimization by showing the evolution of the consecutive poses along the path. The obtained optimized path is depicted in Fig. 7-right.

Scenario II: the query path, shown in Fig. 8-left, connects a starting configuration placed at the lift to a goal configuration that is inside a specific Vacuum Vessel Port Cell both in the TB of ITER. As illustrated in Fig. 8-right, the path optimization behaves well in highly constrained scenarios, fact that is supported by the data presented in Table I, with clear improvements, relative to the query path, on the three performance measures.
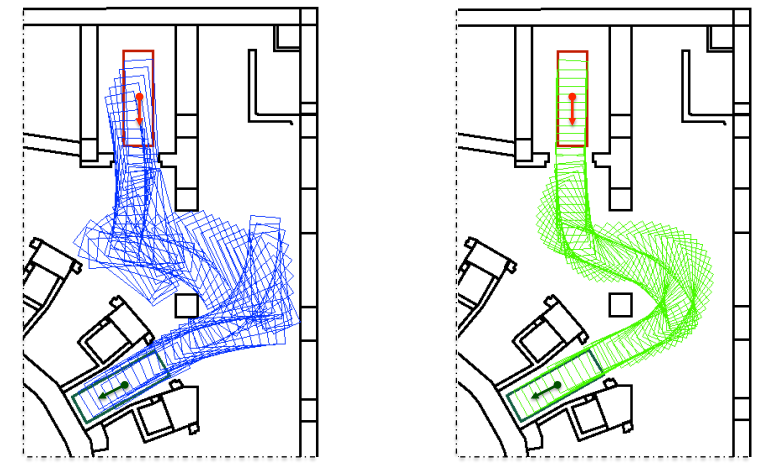

Fig. 8. Path optimization in the TB of ITER: the collision-free query path is not feasible for execution (left); the final optimized path is a feasible and reliable path ensuring a good clearance over the obstacles (right).

Scenario III: this example refers to a CPRHS's parking operation in the HCB of ITER (see Fig. 9-a). The environment is less constrained than the one presented in Fig. 8, but 
is filled with different parked vehicles that play the role of obstacles for the mission purpose. Fig. 9-b illustrates the high maneuverability of the rhombic vehicle but also stresses two important issues of this methodology: (1) The path reaches a state equilibrium that is loosely shaped by the initial query path and hence the optimized path may include unnecessary maneuvers for the achievement of the final goal; (2) The external forces can continuously push the poses, locally stretching the path. This phenomenon makes the optimized path less smooth on some parts and can weaken the condition of a free-collision path (see Fig. 9-c). So far, this issue has been managed by a careful tuning of the spring gain values, $K E$ and $K T$. For each scenario, Table I summarizes the

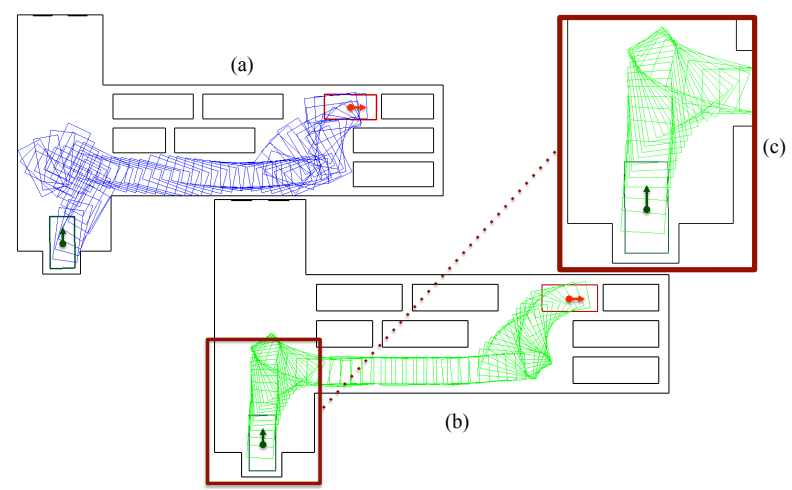

Fig. 9. Parking operation in the HCB of ITER. (a) Provided query path. (b) The optimized solution explores the rhombic capabilities but cannot undo unnecessary maneuvers of the query path. (c) Local stretching of the path.

obtained performance measures for the initial query path (qp) and the evaluated optimized path (op). The optimized paths present an increased clearance, leading to zero the bad clearance, and are shorter and smoother when compared to the rough query path. The results obtained in Scenario III for the rotational component are explained with the growth of this component within the overall vehicle motion during the path optimization. The rough translational motions of the query path grew to smooth and clear rotational motions in the optimized path.

TABLE I

PATH OPTIMIZATION PERFORMANCE

\begin{tabular}{ccccc|cc|cccc} 
& & \multicolumn{4}{c|}{ Clearance } & \multicolumn{2}{c|}{ Length } & \multicolumn{4}{c}{ Smoothness } \\
\cline { 3 - 11 } & & $C$ & $C B$ & $\mu C$ & $L T$ & $L R$ & $\mu S T$ & $\mu S R$ & $\sigma S T$ & $\sigma S R$ \\
\hline \multirow{2}{*}{ I } & qp & 60.58 & 0.22 & 1.55 & 34.67 & 10.27 & 0.91 & 0.27 & 0.36 & 0.95 \\
& op & 81.63 & 0 & 2.09 & 25.27 & 9.29 & 0.66 & 0.25 & 0.03 & 0.97 \\
\hline \multirow{2}{*}{ II } & qp & 39.16 & 4.02 & 0.59 & 53.11 & 6.63 & 0.81 & 0.10 & 0.03 & 0.08 \\
& op & 76.92 & 0 & 1.15 & 37.86 & 3.94 & 0.57 & 0.06 & 0.16 & 0.04 \\
\hline \multirow{2}{*}{ III } & qp & 44.26 & 3.15 & 0.70 & 60.33 & 18.62 & 0.97 & 0.30 & 0.12 & 1.07 \\
& op & 91.09 & 0 & 1.45 & 40.75 & 42.52 & 0.65 & 0.69 & 0.34 & 1.84 \\
\hline
\end{tabular}

\section{CONClusions ANd Future Work}

This paper proposed a rigid-body-spring system as the basis for a new path optimization algorithm, which is intended to work off-line. Departing from the elastic bands approach, this method evades the common approach that formulates paths as particle-systems and path deformation as a pseudo-static simulation. In the proposed approach consecutive poses along the query path are treated as rigid bodies that can move away from obstacles but, at the same time, kept connected due to internal interactions within the overall system. The path deformation leads to a optimization process that continuously shortens and smooths the path while keeping a minimum amount of clearance over the obstacles. The method allows to explicitly handle the vehicle geometric constraints and explores the maneuverability of flexible configurations such as those found in rhombic vehicles, which was an open issue on related studies. Gathered results show the proficiency of this method on evaluating feasible paths in the highly constrained scenarios of ITER while matching the rhombic nature of the vehicle.

Future work will focus on the implementation of a pruning technique to remove the unnecessary maneuvers of the RRT query path. Another further step consists on finding a greedy solution to avoid locally stretching of the path. This can be achieved with a dynamic tuning of the elastic and torsional gains during the path deformation as to achieve a suitable compromise between external and internal interactions.

\section{REFERENCES}

[1] D. Fonte, F. Valente, A. Vale, I. Ribeiro, "A Motion Planning Methodology for Rhombic-like Vehicles for ITER RH Operations", in Proc. of the $7^{\text {th }}$ IFAC Symp. on Intell. Autonomous Vehicles, Italy, 2010.

[2] E. Hairer, C. Lubich, G. Wanner, Geometric numerical integration illustrated by the Stormer Verlet method, 2003, Acta Numerica, vol. 12, pp. $399-450$.

[3] F. Lamiraux, D. Bonnafous, O. Lefebvre, "Reactive path deformation for nonholonomic mobile robots", IEEE Trans. on Rob. and Autom, Dec. 2004, vol. 20, no. 6, pp. 967 - 977.

[4] F.P. Beer, E.R. Johnston, W.E. Clausen, G.H. Staab, Vector Mechanics for Engineers, Dynamics, McGraw-Hill, Hightstown, NJ, 9-th edition; 2009.

[5] H. Kurniawati and T. Fraichard, "From path to trajectory deformation", in Proc. of the IEEE/RSJ Int. Conf. on Intelligent Robots and Systems (IROS), San Diego, USA, Oct. 2007, pp. 159 - 164.

[6] J-C Latombe, Robot Motion Planning, Kluwer Academic Publishers, Boston, MA; 1991.

[7] M.I. Ribeiro, P. Lima, P. Aparício, R. Ferreira, "Conceptual Study on Flexible Guidance and Navigation for ITER Remote Handling Transport Casks". in Proc. of the $17^{\text {th }}$ IEEE NPSS Symp. on Fusion Eng., San Diego, USA, 1997, pp. 969 - 972.

[8] M. Khatib, H. Jaouni, R. Chatila, J.-P. Laumond, "Dynamic path modification for car-like nonholonomic mobile robots", in Proc. of the IEEE Int. Conf. on Robotics and Automation (ICRA), Albuquerque, USA, Apr. 1997, pp. 2920 - 2925.

[9] M.U. Ascher, L.R. Petzold, Computer methods for ordinary differential equations and differential - algebraic equations, Society for Industrial and Applied Mathematics, 1998.

[10] R. Geraerts, M. Overmars, "Clearance Based Path Optimization for Motion Planning", In Proc. of the IEEE ICRA, 2004, pp. 2386 - 2392.

[11] R. Pepy, A.Lambert, H. Mounier, "Path Planning using a Dynamic Vehicle Model”, Infor. and Commun. Technol., 2006, vol. 1, pp. 5376 $-5381$.

[12] R. P. Feynman, R. B. Leighton, M. Sands, The Feynman Lectures on Physics, Addison Wesley, Vol. I, Sec. 9.6; 1963.

[13] S. M. LaValle, J. J. Kuffner, "Rapidly-exploring random trees: Progress and prospects", In B. R. Donald, K. M. Lynch, and D. Rus, editors, Algorithmic and Computational Robotics: New Directions, pp. 293 308, A K Peters, Wellesley, MA; 2001.

[14] S.M. LaValle, Planning Algorithms, Cambridge University Press; 2006.

[15] S. Quinlan, O. Khatib, "Elastic bands: connecting path planning and control", in Proc. of the IEEE ICRA, Atlanta, USA, May 1993, pp. $802-807$.

[16] L.E. Kavraki, P. Svestka, J.-C Latombe, and M.H. Overmars, "Probabilistic roadmaps for path planning in high-dimensional configuration spaces", IEEE Transactions on Robotics and Automation, Aug. 1996, Vol 12, No 4. pp. 566 - 580. 